\title{
Suprachoroidal MIGS Devices
}

\author{
Julian Garcia-Feijoo, Jose Maria Martinez-de-la-Casa, \\ and Lucia Perucho
}

\subsection{Introduction}

The suprachoroidal outflow pathway has the potential to reduce the intraocular pressure (IOP) dramatically, as the pressure gradient between the anterior chamber and the suprachoroidal space/uveal capillaries (colloidal osmotic pressure) permits flow even when IOP is very low. Compared with trabecular bypass minimally invasive glaucoma surgery (MIGS) devices, suprachoroidal MIGS harnesses a pathway that has a much greater IOP-lowering potential as suprachoroidal aqueous drainage is not dependent on the episcleral venous pressure. However, this pathway has a higher risk of severe and prolonged hypotony, because of the greater pressure gradient. The reason that most patients do not develop severe hypotony is because of fibrosis in the suprachoroidal space which restricts aqueous draining from the anterior chamber through the device from exiting the device into the suprachoroidal space. This may limit the long-term success of suprachoroidal devices [1].

\subsection{Physiology of the Suprachoroidal Outflow Pathway}

The natural suprachoroidal outflow pathway drains aqueous from the anterior chamber to the suprachoroidal space via the ciliary muscle [2]. Though the existence of this drainage pathway was proposed more than a century ago, it was poorly understood until monkey studies by Bill et al. in the 1960s allowed its physiology to be

J. Garcia-Feijoo $\cdot$ J. M. Martinez-de-la-Casa $\cdot$ L. Perucho $(\bowtie)$

Department of Ophthalmology, Hospital Clínico San Carlos, Universidad Complutense de Madrid, Madrid, Spain

Instituto de Investigación Sanitaria del Hospital Clínico San Carlos (IdISSC), Madrid, Spain

Cooperative Research Network on Age-Related Ocular Pathology, Visual and Life Quality, Instituto de Salud Carlos III, Madrid, Spain 
better defined [3, 4]. Aqueous permeates through ciliary muscle, the principal site of outflow resistance $[3,5]$, to the supraciliary and suprachoroidal spaces which exert a negative pressure. This outflow is IOP-independent over a wide range of IOP (4-35 mmHg) [3, 6, 7]. Aqueous exits the eye from the suprachoroidal space via two distinct drainage routes: the uveoscleral route (larger molecules: from the suprachoroidal space through the sclera to the orbit) and the uveovortex route (smaller molecules: from the suprachoroidal space to the uveal capillaries and the vortex veins). Of the two drainage routes, the uveovortex drainage pathway is the predominant pathway and is dependent on the difference in the colloid osmotic pressure between the uveal interstitial fluid (low) and the uveal capillaries (high) as well as the intraocular hydrostatic pressure $[3,8]$.

\subsection{Early Surgical Approaches}

It is surgically possible to bypass the ciliary muscle pathway, described above, by disrupting the attachment of the ciliary body to the scleral spur, hence allowing aqueous to flow directly between the anterior chamber and the suprachoroidal space. In 1905, Heine described a technique of cyclodialysis performed ab externo through the sclera using a spatula $[9,10]$. Modifications of the technique were proposed in the twentieth century to prevent closure of the cleft, including the implantation of tissue or other material [11-13].

Unfortunately, these techniques caused significant ocular trauma and their efficacy in lowering the IOP was unpredictable with a high proportion of eyes developing prolonged hypotony followed by significant IOP spikes after spontaneous cleft closure, hence they were abandoned. Moreover, complications including suprachoroidal haemorrhage, hyphaema and secondary cataract were frequent. Nevertheless, this pathway is still unique in its impressive IOP-lowering potential, hence various modifications in the surgical technique of trabeculectomy [14, 15], non-penetrating glaucoma surgery [16] and glaucoma drainage devices [17] have been proposed over the years in vain attempts to utilize suprachoroidal drainage in addition to external filtration. Although these modifications did not result in higher complication rates compared with the conventional surgical techniques, neither did they improve efficacy.

\subsection{Ab-Externo Suprachoroidal Devices}

To avoid the complications associated with excessive filtration, some means of controlling the rate of aqueous outflow to the suprachoroidal space is required. Hence, several ab-externo suprachoroidal devices have been introduced, pioneered by the Gold Glaucoma Shunt (GGS, SOLX Ltd., Waltham, MA, USA) and followed by the STARflo Glaucoma Implant (iSTAR Medical, Isnes, Belgium) and the Aquashunt (OPKO Health Inc., Miami, FL, USA). Despite a sophisticated design incorporating flow control to prevent early hypotony with the GGS, long-term efficacy was poor 
as fibrosis and encapsulation developed around the device [18, 19]. Conjunctival peritomy and scleral flap dissection are also required in order to implant such $a b$ externo suprachoroidal devices, which added to the risk of scarring and, additionally, there was a concern that the GGS might also result in significant corneal endothelial cell loss because of its positioning. Hence, this has led to the development of ab-interno suprachoroidal MIGS devices, which are conjunctiva-sparing and less invasive.

\subsection{Ab-Interno Suprachoroidal MIGS Devices}

The emergence of MIGS has revolutionized glaucoma surgery [20]. The high safety profile of MIGS permits earlier use of surgery in the glaucoma treatment algorithm. $\mathrm{Ab}$ interno suprachoroidal MIGS devices are implanted through a corneal incision, hence spare the conjunctiva. Compared with ab-externo suprachoroidal devices, the potential advantages of accessing the suprachoroidal space with an ab-interno MIGS device are obvious, including less trauma, better safety profile and less inflammation and scarring. However, as with ab-externo suprachoroidal devices, the long-term efficacy of ab-interno devices may also be limited by scarring in the suprachoroidal space.

\subsubsection{CyPass Micro-Stent}

The CyPass Micro-Stent (Alcon Laboratories, Inc., Fort Worth, Texas, USA) was the first commercially available suprachoroidal MIGS device (Fig. 8.1). Originally developed by Transcend Medical, the CyPass Micro-Stent was a 6.35-mm polyamide tube with a 430- $\mu \mathrm{m}$ external diameter and a 300- $\mu \mathrm{m}$ lumen. After implantation into the suprachoidal space, it permitted unrestricted flow between the anterior chamber and the suprachoroidal space. The CyPass Micro-Stent had fenestrations (76- $\mu \mathrm{m}$ pores) along its length, to facilitate additional lateral flow and three retention rings at the proximal end, which acted as reference points for device position during implantation.

\subsubsection{Surgical Technique}

The CyPass Micro-Stent was inserted ab interno into the suprachoroidal space via a clear corneal incision. First, the device was loaded onto the retractable guidewire of the applier, assuming the same curvature as the applier guidewire, thereby facilitating insertion into the suprachoroidal space along the scleral contour. To obtain a good view of the anterior chamber angle, the patient's head was tilted away from the surgeon and the microscope tilted towards the surgeon. The device was inserted via a 20-gauge corneal incision diametrically opposite to the implantation site after pharmacological miosis and filling the target area with cohesive viscoelastic. The goniolens was placed on the cornea and the applier inserted through the corneal incision (Fig. 8.2). The blunt tip of the applier guidewire was slowly advanced 

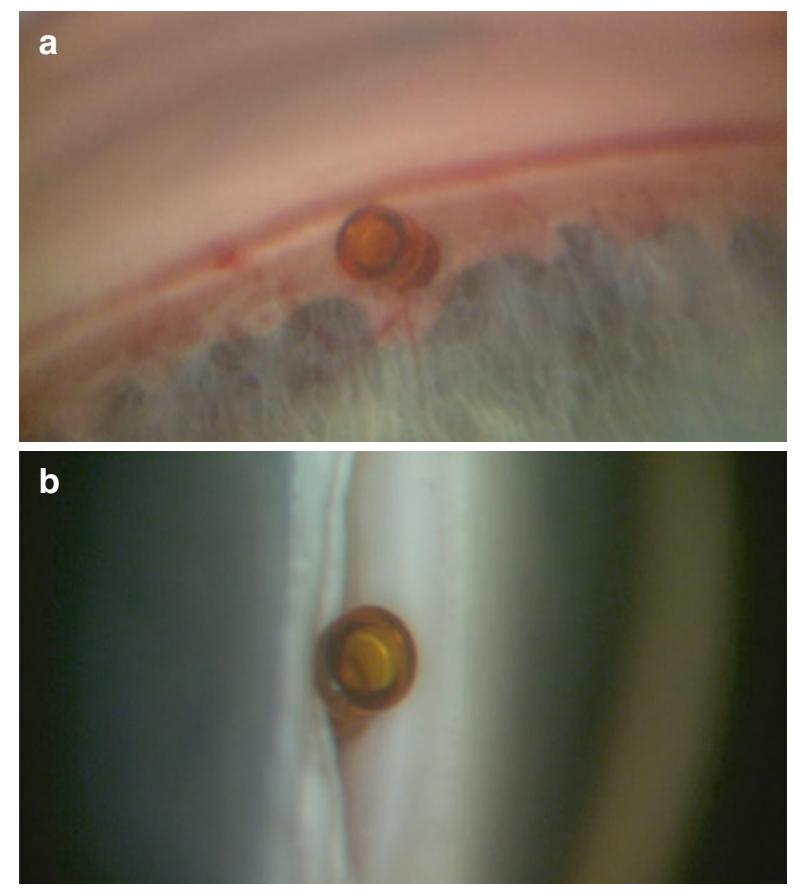

Fig. 8.1 (a) A CyPass Micro-Stent in a good position on gonioscopy in the operating theatre at the end of surgery. Note the blood outlining Schlemm's canal demonstrating that the collar of the CyPass Micro-Stent is sitting at the level of trabecular meshwork, well away from cornea and the entry of the CyPass Micro-Stent into the ciliary body band, peripheral to iris root (copyright Moorfields Eye Hospital and Keith Barton, reproduced with permission). (b) A well-positioned and patent CyPass Micro-Stent visible on gonioscopy a number of weeks after surgery (Copyright Moorfields Eye Hospital and Keith Barton; reproduced with permission)

between scleral spur and ciliary body, ensuring no iris movement when entering the angle. If the guidewire was inserted into the correct tissue plane and, if the insertion angle were correct, very little resistance would be encountered when inserting the device between ciliary body and sclera. The curvature of the applier guidewire permitted the CyPass Micro-Stent to advance along the scleral curvature. Once the CyPass Micro-Stent was at the correct depth, depression of the release button allowed the guidewire to retract. Ideally, the device was positioned so that the rim of the collar was at the upper border of the trabecular meshwork. After implantation of the CyPass Micro-stent, the viscoelastic was removed completely and the corneal incision sealed by hydration. A demonstration of the technique is available online (www.youtube.com/watch?v=_WXNL0CoJws\&list=UUnkpnhwaQCC4Ary7gIyX RIw\&index=2, accessed 2nd November 2019).

\section{Poor Visualization of the Anterior Chamber Angle}

Good visualization of the anterior chamber angle during implantation was important, though less critical than with trabecular meshwork stents as the target 

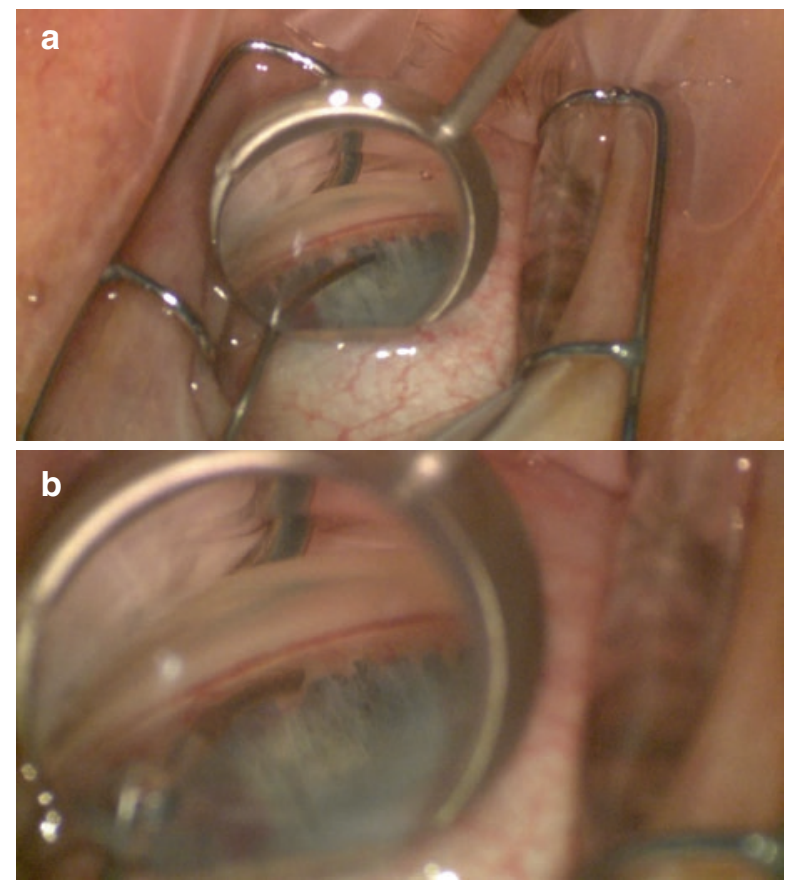

Fig. 8.2 (a, b) Insertion of the CyPass Micro-Stent in Fig. 8.1 (Copyright Moorfields Eye Hospital and Keith Barton; reproduced with permission)

implantation site, the ciliary body band is, on account of its position as the most posterior structure in the angle before the iris root, harder to miss. The CyPass was approved for implantation in eyes with open-angle glaucoma (Shaffer grade 3 or 4).

\section{Resistance Encountered During Implantation}

If resistance was encountered when advancing the device, this was either due to failure of the device to follow the scleral curvature or positioning of the tip of the guidewire in iris or ciliary body rather than between ciliary body and sclera. If the angulation and position of the applier were corrected, the device could be implanted in the suprachoroidal space with very little resistance.

\section{Position of Device Too Anterior}

If the proximal end of the CyPass Micro-Stent was positioned more anterior than the ideal position (rim of the collar at the level of the trabecular meshwork) (Fig. 8.3), the guidewide tube of the applier could be used to gently push the device deeper into the suprachoroidal space.

\section{Position of Device Too Posterior}

If the CyPass Micro-Stent was pushed too far into the suprachoroidal space, then there was a higher risk of post-operative device occlusion by iris. Sometimes the 
Fig. 8.3 (a) Improperly positioned CyPass Micro-Stent protruding anteriorly and abutting cornea 2 years after surgery (copyright Moorfields Eye Hospital and Keith Barton, reproduced with permission). (b) The same CyPass Micro-Stent as (a), demonstrating that three rings are visible and the collar is very close to the cornea on gonioscopy, with a consequent high risk of corneal endothelial cell loss (Copyright Moorfields Eye Hospital and Keith Barton; reproduced with permission)

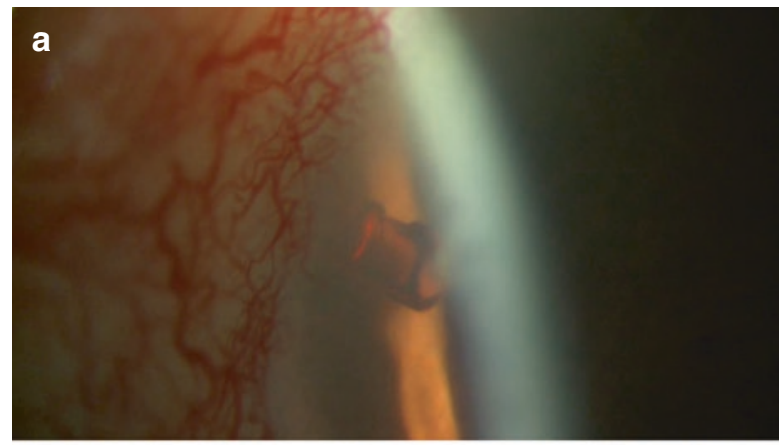

b

device could then be grasped carefully with retinal micro-forceps and pulled gently forwards into the anterior chamber. If the CyPass Micro-Stent were implanted so posteriorly that the tip of its collar could not be visualized, the device would have to be abandoned and left in the supra-choroidal space as attempted removal would cause excessive trauma. Implantation of a second CyPass Micro-Stent in the same eye would then generally be performed at least 2 clock-hours away from the first, in order to avoid the creation of a cyclodialysis cleft between the devices.

\subsubsection{Mechanism of Action}

Ultrasound biomicroscopy [21] and optical coherence tomography (OCT) [22] studies have shown that aqueous accumulated in the suprachoroidal space around and posterior to the CyPass Micro-Stent. In addition, as the device resumed a straight configuration after guidewire retraction, this created a fluid-filled space between the device and the sclera (tenting). Ultrasound biomicroscopy imaging of the suprachoroidal lake has shown that it could extend $360^{\circ}$ circumferentially around the eye after CyPass Micro-Stent implantation. However, the fluid around and posterior to the device, as well as the space between the device and the sclera reduced with time, due to fibrosis in the suprachoroidal space. Given the ease with which choroidal effusions resorb with pressure elevation, it is most likely that aqueous from the suprachoroidal lake exits the eye via the choroidal and vortex venous system. 


\subsubsection{Efficacy}

The safety and efficacy of the CyPass Micro-Stent have been investigated in several clinical studies, including one large randomized controlled trial.

Hoeh et al. [23] reported the 6-month outcomes of combined cataract surgery and CyPass Micro-Stent implantation in an exploratory multi-centre case series of 98 patients. The patients were divided into two groups based on whether their baseline IOP was uncontrolled (IOP $\geq 21 \mathrm{mmHg}$, Cohort 1) or controlled (IOP $<21 \mathrm{mmHg}$, Cohort 2). In uncontrolled patients $(n=57)$, the mean IOP was decreased by $37 \%(p<0.001)$ and the mean number of glaucoma medications was reduced by more than $50 \%(p<0.001)$ at 6 months. Patients whose IOP was controlled at baseline $(n=41)$ had a $71 \%$ reduction in the number of glaucoma medications.

A similar study by Höh et al. [24] reported the 2-year outcomes of combined cataract surgery and CyPass Micro-Stent implantation, with the patients again divided into those with uncontrolled baseline IOP $(n=23$, IOP $\geq 21 \mathrm{mmHg}$, Cohort 1) and those with controlled baseline IOP $(n=59$, IOP $<21 \mathrm{mmHg}$, Cohort 2$)$. The IOP at 24 months was $15.8 \pm 3.8 \mathrm{mmHg}(37 \pm 19 \%$ decrease from baseline IOP) and $16.1 \pm 3.2 \mathrm{mmHg}(0 \pm 28 \%$ decrease from baseline IOP $)$ in Cohort 1 and Cohort 2 , respectively. The mean number of glaucoma medications at 24 months was $1.0 \pm 1.1$ in Cohort 1 and $1.1 \pm 1.1$ in Cohort 2. Fifteen subjects (11\%) required additional incisional glaucoma surgery.

Garcia-Feijoo et al. [25] reported the results of the DUETTE study, a single-arm multi-centre study which examined the efficacy and safety of CyPass Micro-Stent implantation as a solo procedure in patients with medically uncontrolled POAG. Of the 65 patients recruited, 12-month data were available for 55 patients. At 12 months, there was a significant decrease in the mean IOP $(16.4 \pm 5.5 \mathrm{mmHg}$ vs. $24.5 \pm 2.8 \mathrm{mmHg}$, $p<0.001)$ and the mean number of glaucoma medications (1.4 \pm 1.3 vs. $2.2 \pm 1.1$, $p=0.002)$. Nine patients required secondary incisional glaucoma surgery and two patients underwent implantation of a second Cypass Micro-Stent.

Vold et al. [26] reported the 2-year results of the COMPASS study $(n=505)$, a randomized comparative trial comparing stand-alone cataract surgery (control group, $n=131$ ) with combined cataract surgery and CyPass Micro-Stent implantation (microstent group, $n=374)$. At baseline, both groups had similar mean IOP $(24.5 \pm 3.0$ in the control group and $24.4 \pm 2.8 \mathrm{mmHg}$ in the microstent group, $p>0.05$ ) and mean number of medications ( $1.3 \pm 1.0$ in the control group and $1.4 \pm 0.9$ in the microstent group, $p>0.05)$. At 2 years, a greater percentage of patients in the microstent group achieved $\geq 20 \%$ reduction in unmedicated IOP compared with the control group ( $77 \%$ vs. $60 \%, p<0.001$ ). The mean IOP reduction was $7.4 \mathrm{mmHg}$ when combined surgery was performed and $5.4 \mathrm{mmHg}$ when cataract surgery was performed as a standalone procedure $(p<0.001)$. At 2 years, $59 \%$ of the patients in the control group did not require any glaucoma medications compared to $85 \%$ of the patients in the microstent group. Three patients from the microstent group and four patients from the control group required further glaucoma surgery. 


\subsubsection{Complications}

As a minimally invasive and blebless glaucoma surgical procedure, the CyPass Micro-Stent had a better safety profile than conventional glaucoma procedures such as trabeculectomy and tube shunts. The post-operative care was also less intense than that after the above procedures [27]. However, compared with trabecular bypass MIGS procedures, the CyPass Micro-Stent was associated with potentially more severe complications.

\section{Intra-operative Complications}

Serious intraoperative complications were rare with the CyPass. Typically a minor amount of bleeding might occur if the tip of the guidewire engaged the anterior chamber angle in an insufficiently peripheral position, catching the iris root. It was rare for bleeding to impede visualization for implantation but, when it did, injection of additional viscoelastic into the anterior chamber was usually sufficient to improve the view. There was also the possibility of inadvertent lens or corneal damage during implantation.

Significant lateral movement of the applier or an excessively traumatic implantation could have resulted in a cyclodialysis cleft around the CyPass, resulting in chronic postoperative hypotony.

\section{Post-operative Complications}

Inflammation

The incidence of early post-operative inflammation (within the first month) after CyPass Micro-Stent implantation has been reported to be around $4.2-8.6 \%$ and resolved in all cases without any sequelae $[25,26]$. Hoeh et al. observed late-onset inflammation in 3.7\% $(n=5)$ of his patients [28], whereas Kerr et al. reported 10\% $(n=2)$, in theirs [29]. Notably, the two patients with late-onset inflammation reported by Kerr et al. had a history of uveitis.

Eyes with early or late-onset post-operative inflammation require an increased frequency of topical steroids, which can be titrated according to the severity of inflammation and may be necessary for several months. The IOP should be monitored closely in these eyes to identify and treat steroid responsiveness.

\section{Hypotony}

When post-operative hypotony occurred with the Cypass, it was the result of aqueous flow into the suprachoroidal space through the CyPass Micro-Stent or around the device (cyclodialysis cleft).

In the COMPASS study, hypotony occurred in 11 subjects (2.9\%) undergoing combined CyPass Micro-Stent implantation with cataract surgery, with three cases considered clinically significant (i.e. associated with early maculopathy). Hypotony was transient in all 11 subjects and resolved spontaneously. Seven subjects in the microstent group developed a cyclodialysis cleft exceeding $2 \mathrm{~mm}$, but none developed hypotony and did not require re-operation, so clearly were not functional clefts [26]. 
Hoeh et al. observed transient early hypotony in $13.8 \%$ of patients undergoing combined CyPass Micro-Stent implantation and cataract surgery. With the exception of one patient who took 6 months to resolve, the hypotony resolved spontaneously by 1 month [23]. Hoeh et al. also reported early hypotony in $14 \%$ of subjects undergoing combined CyPass Micro-Stent implantation and cataract surgery in another series, with all cases resolving spontaneously without visual sequelae or further surgical intervention [28]. A study reporting 2-year outcomes for CyPass Micro-Stent implantation in conjunction with cataract surgery found a similar incidence of early transient hypotony (15.4\% of eyes), also resolving spontaneously in all cases [24].

When post-operative hypotony occurred after CyPass Micro-Stent implantation, the frequency of topical steroids would be reduced to encourage suprachoroidal fibrosis around the device. Rarely, hypotony persisted or was associated with hypotony maculopathy or choroidal detachment. In such cases, surgical intervention was occasionally required to occlude the Cypass Micro-Stent. Sii et al. [30] have reported two cases of persistent hypotony which were successfully treated by occluding the device's lumen ab interno with a 4-0 Nylon suture (www.youtube. com/watch? $\mathrm{v}=5 \mathrm{zZnrSyB} 5 \mathrm{vM} \&$ list=UUnkpnhwaQCC4Ary7gIyXRIw\&index $=11$ $\& \mathrm{t}=0 \mathrm{~s}$, accessed 2nd November 2019).

The risk of hypotony was believed to be higher in highly myopic eyes.

\section{IOP Spikes}

IOP elevation in the immediate post-operative period (up to $48 \mathrm{~h}$ after the surgery) was most often due to retained viscoelastic and could be remedied by posterior lip pressure on the corneal incision using a hypodermic needle at the slit-lamp or medication. Less commonly, IOP elevation in the immediate post-operative period may have been due to occlusion with blood or iris. An IOP spike after the second or third postoperative week could be due to steroid responsiveness or occlusion of the implant or surrounding cleft with fibrosis (or both).

In the COMPASS study, 16 subjects in the microstent group (4.3\%) developed transient IOP spikes, defined as IOP $\geq 10 \mathrm{mmHg}$ above baseline values. All cases resolved, although three subjects required additional glaucoma surgical intervention for IOP control [26]. The frequency of transient IOP spikes was $10.5 \%$ in a study by Hoeh et al. [23] and $10.8 \%$ in a study by García-Feijoó et al. (defined as IOP $>30 \mathrm{mmHg}$ that resolved either on its own or by adding glaucoma medications) [25]. Kerr et al. reported a higher rate of transient post-operative IOP spikes, occurring in $20 \%$ of subjects. Fortunately, none of these subjects required further glaucoma surgery or experienced a deterioration in best-corrected visual acuity (BCVA) [29].

\section{Hyphaema}

The frequency of post-operative hyphaema was reported at between $1.5 \%$ and $15 \%$, with all cases resolving spontaneously within the first month [24-26, 28, 29]. 
Deterioration in Vision (Loss of $\geq 2$ Lines of BCVA)

After CyPass Micro-Stent implantation, 1.1-3.1\% of patients lost $\geq 2$ lines of BCVA $[25,26,28]$. The causes of the vision loss included cystoid macular oedema, cataract progression (in phakic patients who underwent CyPass Micro-Stent implantation as a solo procedure), corneal oedema or posterior capsular opacification, with the management directed at each cause (e.g. cataract surgery, YAG laser capsulotomy).

\section{Device Occlusion}

Occlusion of the CyPass Micro-Stent with peripheral anterior synechiae (PAS) occurred in $2.1 \%$ of subjects in the COMPASS study [26]. García-Feijoó et al. reported that the device was occluded by PAS in two subjects $(3.1 \%)$, and Nd:YAG laser was successfully used to clear the occlusion in one subject [25]. Hoeh et al. reported partial or complete device obstruction in nine subjects $(5.4 \%)$, of which the device was occluded by PAS in two [28]. They also reported that occlusion occurred within the first 3 months in $80 \%$ of the 12 subjects $(8.8 \%)$ in whom the CyPass Micro-Stent was occluded and was usually due to excessively posterior implantation of the device [24].

\section{Device Malposition}

Device malposition occurred in two patients and device migration/dislodgement occurred in two patients in the COMPASS study [26]. Hoeh et al. reported that one subject with an anteriorly positioned CyPass Micro-Stent required additional surgery to push the device further into the suprachoroidal space [23]. Kerr et al. reported that, in the hands of an experienced surgeon, device re-positioning can easily be performed at the slit lamp with a 30-gauge needle [29]. If the position of the CyPass Micro-Stent was too anterior after the surgery, it should be re-positioned as soon as possible before fibrosis and encapsulation develops.

Conversely, if the CyPass Micro-Stent was implanted too posteriorly in the suprachoroidal space, there was a higher risk of device occlusion by iris or PAS [28].

\section{Additional Glaucoma Surgery}

In the COMPASS study, three subjects $(0.8 \%)$ in the microstent group required additional glaucoma surgery to control the IOP [26]. A higher glaucoma re-operation rate was reported by García-Feijoó et al., with 11 subjects (16.9\%) requiring additional glaucoma surgery, mostly within the first 6 months. A second CyPass MicroStent was implanted in two subjects and the remaining nine subjects required subsequent trabeculectomy [25]. Hoeh et al. also reported that additional glaucoma surgery was required in $11.0 \%$ [15] subjects [28]. The COMPASS study included subjects with mild to moderate POAG on $1.4 \pm 0.9$ glaucoma medications at baseline, many of whom were medically controlled prior to medication washout [26]. On the other hand, in the study by Garcia-Feijoo et al., the mean number of glaucoma medicines at baseline was $2.2 \pm 1.1$, and all subjects were medically uncontrolled [25]. 


\section{Corneal Endothelial Cell Loss and Corneal Decompensation}

The COMPASS-XT study (a post-approval extension of the randomized clinical COMPASS trial) showed that patients who had undergone combined CyPass MicroStent implantation with phacoemulsification had a significantly greater reduction in endothelial cell counts than patients who had phacoemulsification alone, 5 years after surgery. Based on these findings, Alcon, the manufacturer, voluntarily withdrew the CyPass Micro-Stent from the global market in August 2018 (www.alcon. com/cypass-recall-information). The extent of endothelial cell loss in the COMPASS-XT study correlated with the number of retention rings visible on gonioscopy, hence the associated corneal damage was almost certainly a consequence of the device position in the angle. Endothelial cell loss was more prominent when two or more retention rings were visible in the anterior chamber. Though none of the patients developed clinically evident corneal decompensation, this was of concern because the US Food and Drug Administration (FDA) restricts the use of the CyPass Micro-Stent to adult patients with mild-to-moderate open-angle glaucoma in conjunction with cataract surgery. The safety of the device is particularly important in this group of patients who are conventionally treated with glaucoma medication. If the CyPass Micro-Stent should become available again in the future, it is likely that the manufacturer's directions on the surgical implantation technique would be amended to recommend a more posterior positioning, specifying that the device should not protrude, ideally above the trabecular meshwork or at worst, the Schwalbe's line. It would be less likely for significant endothelial cell loss to occur in these circumstances. Alcon may also consider extending the indication for the CyPass Micro-Stent to more refractory cases of glaucoma.

In an earlier study, Hoeh $\mathrm{H}$ et al. reported that the incidence of contact between the CyPass Micro-Stent and corneal endothelium was $1.2 \%$, as a consequence of anterior device placement. None of these subjects experienced visual loss or required additional surgery, albeit with a short follow-up of $294 \pm 121$ days [28]. In another study by Höh $\mathrm{H}$ et al., device-corneal endothelial contact occurred in 3.7\% of subjects [24].

If the CyPass Micro-Stent was positioned too anteriorly or if contact is detected between the device and the corneal endothelium, it was advisable to re-position the device in the early postoperative period. Within a few weeks, fibrosis develops around the device, preventing it from being easily re-positioned or removed. In such circumstances, the CyPass Micro-Stent could be trimmed using 23 gauge vitrectomy scissors, so that it does not protrude beyond the Schwalbe's line (www.youtube.com/watch? $\mathrm{v}=\mathrm{mRH}$ dplofoBM\&list=UUnkpnhwaQCC4Ary7gIyXRIw\&inde $\mathrm{x}=5 \& \mathrm{t}=0 \mathrm{~s}$, accessed $2 \mathrm{nd}$ November 2019).

\section{Cataract Progression}

In a multicentre, single-arm interventional study by Garcia-Feijoo et al., cataract progression occurred in $12.2 \%$ of phakic eyes 1 year after stand-alone CyPass Micro-Stent implantation [25]. Höh et al. reported that CyPass Micro-Stent implantation was associated with cataract progression in $2 \%$ of phakic eyes [24]. 


\subsection{2 iStent Supra}

The iStent Supra (Glaukos Corporation, San Clemente, CA, USA) is another $a b$ interno suprachoroidal MIGS device which has the Conformité Européene Mark (Fig. 8.4). It is a ridged curved tube made of heparin-coated polyethersulfone and titanium. The length of the iStent Supra is $4 \mathrm{~mm}$, with an interior lumen diameter of $165 \mu \mathrm{m}$. The mechanism of action of the iStent Supra is very similar to that of the CyPass Micro-Stent.

\subsubsection{Implantation Technique}

The implantation technique of the iStent Supra is similar to that described above for the CyPass Micro-Stent. A 1.5-mm clear corneal incision is sufficient for the insertion of the iStent Supra.

\subsubsection{Efficacy and Safety}

Myers et al. [31] reported the efficacy of iStent Supra implantation combined with the implantation of two iStent Trabecular Micro-Bypass Stents and post-operative travoprost in patients with refractory open-angle glaucoma and previously failed glaucoma filtration surgeries. This case series reported the 4-year outcomes although it was originally designed to be a 5-year study. The mean unmedicated IOP at all visits was $\leq 13.7 \mathrm{mmHg}$ ( $\geq 37 \%$ reduction from baseline). Among eyes without additional medication or surgery, $\geq 91 \%$ of eyes had $\geq 20 \%$ decrease in IOP on one medication compared with pre-operative medicated IOP at all post-operative visits. At 4 years, $97 \%$ and $98 \%$ of the eyes achieved IOP $\leq 15$ and $\leq 18 \mathrm{mmHg}$ respectively on one medication. Additional medication was required in six eyes, and none of the patients required additional glaucoma surgery. The most frequent adverse event was cataract progression (16\% of the subjects) [31].

Fig. 8.4 iStent Supra (Copyright Glaukos Corporation,

SanClemente, CA, USA; reproduced with permission)

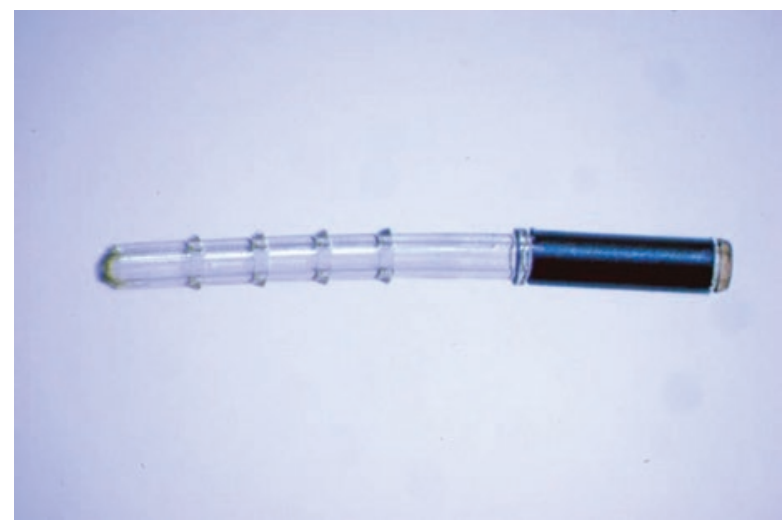




\subsubsection{MINIject}

The MINIject (iSTAR Medical, Isnes, Belgium) is the latest suprachoroidal MIGS device to be introduced (Fig. 8.5). Like its predecessor, the STARflo Glaucoma Implant (iSTAR Medical, Isnes, Belgium), the MINIject is made from STAR ${ }^{\circledR}$ material, which comprises of soft and flexible medical-grade silicone that conforms to the curvature of the eye. The STAR ${ }^{\circledR}$ material is composed of an organized network of hollow spheres with a micro-porous, multi-channel matrix which promotes biointegration of surrounding tissues into the material, with the intention of reducing fibrosis and scarring, hence increasing the efficacy of the device. The MINIject is $5 \mathrm{~mm}$ in length and the green ring at the anterior segment of the device is used as a reference point for device position during implantation.

\subsubsection{Implantation Technique}

The implantation technique of the MINIject is similar to that described above for the CyPass Micro-Stent. The MINIject implant is preloaded in a transparent sheath attached to an applier handle, and sliding a wheel on the applier handle retracts the sheath back into the handle, leaving the device in place in the suprachoroidal space. Correct placement depth is achieved when the green ring is at the level of the scleral spur.

\subsubsection{Efficacy and Safety}

The first-in-human STAR-I trial for stand-alone MINIject implantation (ClinicalTrials.gov Identifier: NCT03193736) included 25 patients with mild-to-moderate POAG uncontrolled by topical glaucoma medications. Six-month data from the STAR-I trial showed a reduction in the mean \pm standard error IOP $(23.2 \pm 0.6$ vs. $14.2 \pm 0.9, p<0.0001)$ and the mean \pm standard deviation number of glaucoma medications $(2.0 \pm 1.1$ versus $0.3 \pm 0.7)$, with 21 patients $(87.5 \%)$ being medicationfree and 23 patients $(95.8 \%)$ achieving a minimum 20\% IOP reduction from baseline. There were no serious adverse events related to the device or procedure and no additional glaucoma surgery was required. The mean central or peripheral corneal endothelial cell density was not significantly different from baseline [32]. At the time of writing, the 1-year data from the STAR-I trial have not been published.

Fig. 8.5 MINIject (Copyright iSTAR Medical, Isnes, Belgium; reproduced with permission)

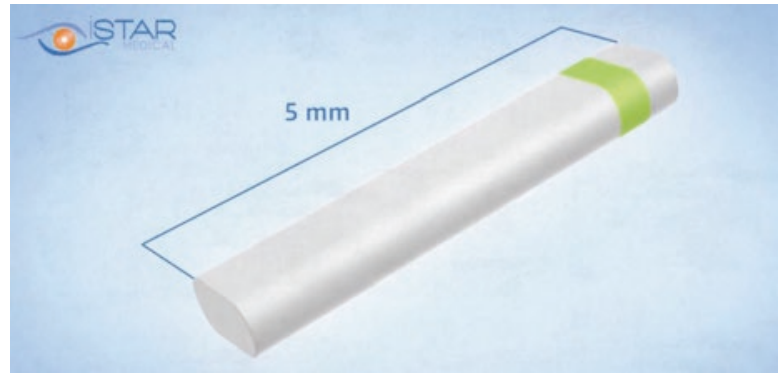




\section{References}

1. Bakharev AV, Fedorov AA, Batmanov LE. Comparative experimental morphological study of the impact of various drainages for cyclodialysis to adjacent tissues. Vestn oftalmol. 2008;12(2):44-6.

2. Johnson M, McLaren JW, Overby DR. Unconventional aqueous humor outflow: a review. Exp Eye Res. 2017;158:94-111.

3. Bill A, Phillips CI. Uveoscleral drainage of aqueous humor in human eyes. Exp Eye Res. 1971;12:275-81.

4. Bill A. The aqueous humor drainage mechanism in the cynomolgus monkey (Macaca irus) with evidence for unconventional routes. Investig Ophthalmol. 1965;4:911-9.

5. Alm A, Nilsson SFE. Uveoscleral outflow-a review. Exp Eye Res. 2009;88(4):760-8.

6. Bill A. Some thoughts on the pressure dependence of uveoscleral flow. J Glaucoma. 2003;12(1):88-9.

7. Yablonski ME. Some thoughts on the pressure dependence of uveoscleral flow. J Glaucoma. 2003;12(1):90-2.

8. Pederson JE, Gaasterland DE, et al. Uveoscleral aqueous outflow in the rhesus monkey: importance of uveal reabsorption. Investig Ophthalmol Vis Sci. 1977;16:1008-17.

9. Böke H. History of cyclodialysis. In memory of Leopold Heine 1870-1940. Klin Monbl Augenheikd. 1990;197(4):340-8.

10. Knapp A. The operative treatment of glaucoma by cyclodialysis. JAMA. 1909;53(10):765-7.

11. Barkan O, Boyle SF, Maisner S. On the surgery of glaucoma: mode of action of cyclodialysis. Cal West Med. 1936;44(1):12-6.

12. Barkan O. Cyclodialysis, multiple or single, with air injection; an operative technique for chronic glaucoma. Am J Ophthalmol. 1947;30(9):1063-73.

13. Jordan JF, Engels BF, Dinslage S, et al. A novel approach to suprachoroidal drainage for the surgical treatment of intractable glaucoma. J Glaucoma. 2006;15(3):200-5.

14. Lázaro García C, Benítez del Castillo JM, Castillo Gómez A, García Feijoó J, Macías Benítez JM, García Sánchez J. Lens fluorophotometry following trabeculectomy in primary open angle glaucoma. Ophthalmology. 2002;109:76-9.

15. Gupt S, Gupta V. Trabeculectomy augmented with ciclodialysis: a surgical option for refractory Glaucomas. J Glaucoma. 2016;25(7):e726.

16. Muñoz G. Nonstich suprachoroidal technique for T-Flux implantation in deep sclerectomy. $\mathrm{J}$ Glaucoma. 2009;18(3):262-4.

17. Ozdamar A, Aras C, Karacorlu M. Suprachoroidal seton implantation in refractory glaucoma: a novel surgical technique. J Glaucoma. 2003;12:354-9.

18. Hueber A, Roters S, Jordan JF, Konen W. Retrospective analysis of the success and safety of Gold Micro Shunt Implantation in glaucoma. BMC Ophthalmol. 2013;13:35.

19. Figus M, Lazzeri S, Fogagnolo P, et al. Supraciliary shunt in refractory glaucoma. Br J Ophthalmol. 2011;95:1537-41.

20. Saheb H, Ahmed II. Micro-invasive glaucoma surgery: current perspectives and future directions. Curr Opin Ophthalmol. 2012;23(2):96-104.

21. Gonzalez-Pastor E, et al. UBM findings after suprachoroidal CyPass implant for glaucoma: one year follow-up. ARVO; 2013.

22. Saheb H, Ianchulev T, Ahmed II. Optical coherence tomography of the suprachoroid after CyPass Micro-Stent implantation for the treatment of open-angle glaucoma. Br J Ophthalmol. 2014;98:19-23.

23. Hoeh H, Ahmed II, Grisanti S, Grisanti S, Grabner G, Nguyen QH, Rau M, Yoo S, Ianchulev T. Early postoperative safety and surgical outcomes after implantation of a suprachoroidal micro-stent for the treatment of open-angle glaucoma concomitant with cataract surgery. J Cataract Refract Surg. 2013;39(3):431-7. 
24. Höeh H, Grisanti S, Grisanti S, Rau M, Ianchulev S. Two-year clinical experience with the CyPass micro-stent: safety and surgical outcomes of a novel supraciliary micro-stent. Klin Monatsbl Augenheilkd. 2014;231(4):377-81.

25. García-Feijoó J, Rau M, Grisanti S, et al. Supraciliary micro-stent implantation for open-angle glaucoma failing topical therapy: 1 year results of a multicenter study. Am J Ophthalmol. 2015;159:1075-81.

26. Vold S, Ahmed II, Craven ER, et al. Two-year COMPASS trial results: supraciliary microstenting with phacoemulsification in patients with open-angle glaucoma and cataracts. Ophthalmology. 2016;123(10):2103-12.

27. Gedde SJ, Herndon LW, Brandt JD, et al. Postoperative complications in the Tube Versus Trabeculectomy (TVT) study during five years of follow-up. Am J Ophthalmol. 2012;153:804-14.

28. Hoeh H, Vold SD, Ahmed IK, et al. Initial clinical experience with the CyPass micro-stent: safety and surgical outcomes of a novel supraciliary microstent. J Glaucoma. 2016;25:106-12.

29. Kerr NM, Wang J, Perucho L, Barton K. The safety and efficacy of supraciliary stenting following failed glaucoma surgery. Am J Ophthalmol. 2018;190:191-6.

30. Sii S, Triolo G, Barton K. Case series of hypotony maculopathy after CyPass insertion treated with intra-luminal suture occlusion. Clin Exp Ophthalmol. 2019;47(5):679-80.

31. Myers JS, Masood I, Hornbeak DM, et al. Prospective evaluation of two iStent ${ }^{\circledR}$ trabecular stents, one iStent SUPRA ${ }^{\circledR}$ suprachoroidal stent, and postoperative prostaglandin in refractory glaucoma: 4-year outcomes. Adv Ther. 2018;35(3):395-407.

32. Denis P, Hirneib C, Reddy KP, et al. A first-in-human study of the efficacy and safety of MINIject in patients with medically uncontrolled open-angle glaucoma (STAR-I). Ophthalmol Glaucoma. 2019;2(5):290-7.

Open Access This chapter is licensed under the terms of the Creative Commons Attribution 4.0 International License (http://creativecommons.org/licenses/by/4.0/), which permits use, sharing, adaptation, distribution and reproduction in any medium or format, as long as you give appropriate credit to the original author(s) and the source, provide a link to the Creative Commons license and indicate if changes were made.

The images or other third party material in this chapter are included in the chapter's Creative Commons license, unless indicated otherwise in a credit line to the material. If material is not included in the chapter's Creative Commons license and your intended use is not permitted by statutory regulation or exceeds the permitted use, you will need to obtain permission directly from the copyright holder.

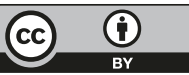

